\title{
Tributo a los médicos hondureños fallecidos durante la pandemia de COVID-19, octubre 2020
}

\author{
Tribute to fallen Honduran physicians during the COVID-19 pandemic, October 2020
}

\author{
Jackeline Alger, ${ }^{1,2}$ Flor Mejía, ${ }^{1,3}$ Melissa Mejía., \\ ${ }^{1}$ Consejo Editorial, Revista Médica Hondureña, Colegio Médico de Honduras. \\ ${ }^{2}$ Médica, Doctorado (PhD) en Parasitología; Unidad de Investigación Científica, Facultad de Ciencias Médicas (FCM), Universidad Nacional Autónoma de Honduras \\ (UNAH); Departamento de Laboratorio Clínico, Hospital Escuela Universitario. \\ ${ }^{3}$ Licenciada en Pedagogía, Centro de Educación Médica Continua, Colegio Médico de Honduras. \\ ${ }^{4}$ Médica Especialista en Medicina Física y Rehabilitación; Secretaría de Asuntos Educativos y Culturales, Colegio Médico de Honduras; Tegucigalpa.
}

El Aniversario Nonagésimo de publicación ininterrumpida de la Revista Médica Hondureña lo estamos conmemorando en el año 2020, año de la pandemia de COVID-19. ${ }^{1}$ Este tiempo de pandemia es un período difícil para toda la humanidad y de un alto impacto para el personal de salud a nivel global, con efectos negativos sobre el bienestar general y aumento en la mortalidad. ${ }^{2,3}$ En Honduras, el personal de salud también ha sufrido estas consecuencias y a octubre de 2020 el Colegio Médico de Honduras ha informado la muerte de 61 colegas durante la pandemia (Cuadro 1).

La infección producida por el nuevo coronavirus SARSCoV-2 y originada en China en diciembre de 2019, fue declarada como pandemia por la Organización Mundial de la Salud en marzo de 2020. ${ }^{4}$ Siete meses después, a finales del mes de octubre, se han informado más de 43 millones de casos y más de un millón de muertes en todo el mundo. ${ }^{5}$ En vista de que existe una subestimación del número total de casos de COVID-19, debido a que solamente una fracción de los mismos es confirmada mediante pruebas de laboratorio y por la alta proporción de casos asintomáticos o subclínicos, también existe una subestimación en el número de muertes. La mortalidad es una de las formas más importantes de medir la carga de una enfermedad. Existen varias plataformas digitales que presentan diferentes análisis de la mortalidad por COVID-19 por país y a nivel global. ${ }^{5,6}$ Las diferencias en la mortalidad por país pueden deberse, entre otros factores aún no reconocidos, al número de pruebas de laboratorio aplicadas, a la edad de la población y a las características del sistema de salud. ${ }^{6}$

En Honduras, los primeros casos de COVID-19 se informaron el 10 de marzo de $2020 .{ }^{7} \mathrm{Al} 26$ de octubre se habían informado 93,214 casos y 2,623 muertes. ${ }^{5}$ El estimado de 263 muertes por millón de habitantes de Honduras es similar al informado en la misma fecha por Canadá (264) y superior al

Recibido: 07-08-2020; Aceptado para publicación 31-10-2002

Dirección para correspondencia: Dra. Melissa Mejía G.

Correo electrónico: cenemec@colegiomedico.hn

Declaración de relaciones y actividades financieras y no financieras y conflictos de interés: ninguna.

DOI: https://doi.org/10.5377/rmh.v88iSupl.1.11682 informado por Costa Rica (257), República Dominicana (204), Guatemala (203) y El Salvador (147); todos ellos con un número de pruebas de laboratorio realizadas por millón de habitantes que varía desde 1.02 a 11.45 veces más que el número realizado en Honduras. ${ }^{5}$ Estos datos de muerte por COVID-19 en Honduras, los más altos de Centro América y República Dominicana, después de Panamá (608), podrían explicarse por algunas de las características del país que ya se han descrito en otras epidemias como la del dengue en el año 2019 y que se destacan por su aplicación y relevancia en el contexto actual de la pandemia de COVID-19.8,9

Honduras afronta determinantes sociales y sanitarias que requieren atención urgente e integral. Entre las determinantes sociales se encuentran la pobreza y la desigualdad (índice Gini de 0.52), educación insuficiente, migración masiva, criminalidad, altas tasas de homicidio y narcotráfico. ${ }^{10}$ Entre los determinantes sanitarios se pueden señalar el crecimiento desorganizado de la población, la urbanización no planificada, el acceso insuficiente al agua segura, el acceso limitado a una infraestructura sanitaria deteriorada e insuficiente y las consecuencias ecológicas del cambio climático. Se suman los efectos negativos de la gobernanza y las políticas sanitarias fallidas, la privatización del sistema de salud y la corrupción, con desvío de fondos públicos millonarios como una práctica gubernamental generalizada y el consecuente debilitamiento de los principales programas de salud y de educación. ${ }^{8,9} \mathrm{Y}$, sin embargo, a pesar de las necesidades evidentes y apremiantes, el gasto militar de Honduras ha aumentado constantemente desde el año 2010.11 Todas estas características del país se han constituido en elementos potenciadores del impacto de la pandemia sobre la salud pero también sobre la sociedad en general y la economía, y sus efectos negativos a mediano y largo plazo se encuentran en desarrollo en toda la región. ${ }^{12}$

Desde los primeros meses del año 2020 se alertó a nivel global acerca de los efectos negativos de la pandemia sobre el personal de salud y la sobrecarga al sistema sanitario. ${ }^{13-17}$ Se destacan en estas comunicaciones, los editoriales, opiniones y puntos de vista acerca de la importancia de la protección del 
Cuadro 1. Listado de médicos fallecidos durante la pandemia de COVID-19, Colegio Médico de Honduras, 10 de marzo a 31 de octubre de 2020.

\begin{tabular}{|c|c|c|c|}
\hline No. & NOMBRE & $\begin{array}{l}\text { MEDICINA GENERAL / } \\
\text { ESPECIALIDAD }\end{array}$ & $\begin{array}{l}\text { FECHA DEL } \\
\text { DECESO }\end{array}$ \\
\hline 1 & Virginia A. Figueroa Girón & Dermatología & $13 / 03 / 2020$ \\
\hline 2 & América Peñalva David & Master en Salud Pùblica & $16 / 03 / 2020$ \\
\hline 3 & Miguel Omar Pavón & Medicina General & $20 / 03 / 2020$ \\
\hline 4 & Ernesto Argueta Ariza & Cardiologia & $23 / 03 / 2020$ \\
\hline 5 & Sagrario Luna Zelaya & Medicina General & $30 / 03 / 2020$ \\
\hline 6 & Denise Roxana Murillo Martínez & Medicina General & $31 / 03 / 2020$ \\
\hline 7 & José Modesto Meza Palma & Psiquiatría & $11 / 04 / 2020$ \\
\hline 8 & Jesús Américo Reyes Ticas & Psiquiatría & $21 / 04 / 2020$ \\
\hline 9 & Oscar Rolando Durón Durán & Medicina General & $24 / 04 / 2020$ \\
\hline 10 & Karol Lizeth Córdova Burgos & Medicina General & $23 / 05 / 2020$ \\
\hline 11 & Glenda Yamile Mejía Petit & Medicina General & $04 / 06 / 2020$ \\
\hline 12 & Alexis Javier Reyes Amaya & Medicina General & $19 / 06 / 2020$ \\
\hline 13 & Nasry Kamal Handal Handal & Pediatría & $19 / 06 / 2020$ \\
\hline 14 & Mario Benítez & Radiología & $20 / 06 / 2020$ \\
\hline 15 & Leónidas Romero Rodríguez & Medicina Interna, Neumología & $21 / 06 / 2020$ \\
\hline 16 & Héctor Ramón Paz Rivera & Medicina General & $26 / 06 / 2020$ \\
\hline 17 & Carlene Annette Hurst & Medicina General & $28 / 06 / 2020$ \\
\hline 18 & Fidelia Odeth Andonie & Medicina General & $29 / 06 / 2020$ \\
\hline 19 & Mario Pavón Moncada & Ginecologia y Obstetricia & $30 / 06 / 2020$ \\
\hline 20 & Iris Ondina Gallegos Lambur & Medicina General & $01 / 07 / 2020$ \\
\hline 21 & Luis Antonio Reyes Jerezano & Medicina General & $04 / 07 / 2020$ \\
\hline 22 & Héctor Baltazar Hernández Meza & Medicina General & $05 / 07 / 2020$ \\
\hline 23 & Marvin Antonio Yánez & Medicina General & $07 / 07 / 2020$ \\
\hline 24 & Yamilette Jackeline Morales Duarte & Medicina General & $08 / 07 / 2020$ \\
\hline 25 & Gustavo Francisco Carías Sarmiento & Ginecología y Obstetricia & $08 / 07 / 2020$ \\
\hline 26 & Marlon Javier Aviléz Rápalo & Medicina General & $10 / 07 / 2020$ \\
\hline 27 & Carlos Yamil Maradiaga & Medicina General & $11 / 07 / 2020$ \\
\hline 28 & Saúl Antonio Mayorquín Enríquez & Medicina General & $12 / 07 / 2020$ \\
\hline 29 & Oscar Arturo Ney Rivera & Pediatría & $14 / 07 / 2020$ \\
\hline 30 & José Adalberto Mejía & Medicina General & $15 / 07 / 2020$ \\
\hline 31 & Merlin Fernández Rápalo & Salud Pública & $18 / 07 / 2020$ \\
\hline 32 & Rubén Darío Casanova & Urología & $20 / 07 / 2020$ \\
\hline 33 & German Francisco Gallardo Lopez & Medicina General & $25 / 07 / 2020$ \\
\hline 34 & José de Jesús Martínez Benítez & Medicina General & $27 / 07 / 2020$ \\
\hline 35 & Patricia María Padgett Campos & Medicina General & $29 / 07 / 2020$ \\
\hline 36 & Tomas Alberto Cáceres Castro & Salud Ocupacional & $29 / 07 / 2020$ \\
\hline 37 & German Mauricio Erazo Santos & Ginecología, Master en Salud Pública & $31 / 07 / 2020$ \\
\hline 38 & Juan René Valladares Lemaire & Neurocirugía & $02 / 08 / 2020$ \\
\hline 39 & Pablo Enrique Ulloa Cáceres & Maestría en Epidemiología & 05/08/2020 \\
\hline 40 & Obdulio García Carías & Pediatría & 05/08/2020 \\
\hline 41 & Alfredo Oseguera Licona & Medicina General & 08/08/2020 \\
\hline 42 & Juan Ismael Cálix Ardón & Medicina General & $12 / 08 / 2020$ \\
\hline 43 & Manuel Antonio Ordóñez Gallardo & Medicina General & $18 / 08 / 2020$ \\
\hline 44 & Luis Alonso Guardado González & Medicina General & $19 / 08 / 2020$ \\
\hline 45 & Héctor Raúl Gómez Hiza & Medicina General & $21 / 08 / 2020$ \\
\hline 46 & Reina Iris Bonilla Moreno & Medicina General & $22 / 08 / 2020$ \\
\hline 47 & Ada Ester Velásquez Romero & Medicina General & $23 / 08 / 2020$ \\
\hline 48 & Ronald Ricardo Lagos Mejía & Medicina General & $24 / 08 / 2020$ \\
\hline 49 & Edwin Kilmer Del Cid Vásquez & Medicina General & 02/09/2020 \\
\hline 50 & Jesús Fernando Montoya Girón & Medicina General & $16 / 09 / 2020$ \\
\hline 51 & Manuel Sarmiento Rodríguez & Medicina General & $25 / 09 / 2020$ \\
\hline 52 & Job Gerardo Villanueva Núñez & Medicina General & $30 / 09 / 2020$ \\
\hline 53 & Arnoldo Salvador Carranza Melgares & Medicina General & $01 / 10 / 2020$ \\
\hline 54 & Héctor Eduardo Dubón Fuentes & Medicina General & $12 / 10 / 2020$ \\
\hline 55 & Juan Carlos García Zambrano & Medicina General & $18 / 10 / 2020$ \\
\hline 56 & Donaldo Villalobos Martínez & Medicina General & $20 / 10 / 2020$ \\
\hline 57 & Luis Antonio Enamorado Vaquero & Medicina Interna & $20 / 10 / 2020$ \\
\hline 58 & Wilberto Lagos Lagos & Pediatría & $24 / 10 / 2020$ \\
\hline 59 & Enrique Adolfo Cruz Maradiaga & Otorrinolaringología & $25 / 10 / 2020$ \\
\hline 60 & Adalid Humberto Ávila Argueta & Medicina General & $27 / 10 / 2020$ \\
\hline 61 & Mercedes Alexandra Álvarez Álvarez & Medicina General & $29 / 10 / 2020$ \\
\hline
\end{tabular}

personal de salud. A diferencia de la población general, el personal de salud no puede quedarse en casa ni guardar distancia en la atención a los pacientes; por el contrario, debe asumir responsablemente un riesgo de contagio con los consecuentes resultados negativos ya conocidos. En particular, uno de estos editoriales resalta que los trabajadores de la salud, a diferencia de los ventiladores o los hospitales móviles, no pueden ser fabricados urgentemente o desempeñarse al $100 \%$ de rendimiento por períodos prolongados. ${ }^{17}$ Por lo tanto, es de vital importancia que la población, las instituciones y los gobiernos contribuyan a garantizar las mejores condiciones para su buen desempeño y seguridad. La provisión adecuada y oportuna del equipo de protección personal es una medida importante que debe ser complementada con otras medidas incluyendo aquellas que la población debe implementar al quedarse en casa y limitar su circulación a lo estrictamente necesario, la cancelación de eventos no esenciales, el suministro oportuno $y$ adecuado de otros insumos institucionales como agua, jabón, papel toalla y gel de manos; así como también descanso, apoyo psicológico y apoyo familiar. De manera importante, se debe contar con los procedimientos operativos estándar, actualizados con la mejor evidencia disponible, para el desarrollo de las funciones institucionales asistenciales, académicas y administrativas. Es una realidad que el bienestar y seguridad del personal de salud se traduce en el bienestar y seguridad de los pacientes, tal y como recientemente fue destacado por la Organización Mundial de la Salud. ${ }^{18}$ Todas estas acciones conllevan el propósito de facilitar al personal de salud aliviar el sufrimiento y salvar vidas.

El 22 de octubre de 2020, la Junta Directiva del Colegio Médico de Honduras realizó un homenaje a los médicos fallecidos durante la pandemia y a aquellos colegas que se encuentran en la primera línea de atención. Durante el evento que se realizó en las instalaciones del Colegio Médico de Honduras en Tegucigalpa, y que se transmitió por las redes sociales, se colocaron fotografías de cada médico fallecido en báculos de madera en el área verde frontal donde se sembraron árboles cipreses y se colocó un letrero con la leyenda "Estos árboles nos darán el oxígeno que les faltó a nuestros colegas para seguir viviendo y nos darán el oxígeno que necesitamos para continuar la lucha por un sistema de salud público, justo $y$ 
de calidad para el pueblo hondureño." Emblemáticamente, se encendieron antorchas y se develó un monumento representando al médico con vestimenta de trabajo, gabacha, gorra y mascarilla, con el pie izquierdo descalzo simbolizando la falta de equipo de protección personal e insumos, sosteniendo un corazón en la mano izquierda simbolizando el compromiso y pasión con la que el médico realiza su trabajo y la mano derecha en forma de la rama de un árbol simbolizando la esperanza y la vida. Al fondo, en la fachada del edificio principal, con vista al monumento conmemorativo, un mural representa con vistosos colores y formas, la ardua labor que realizan los médicos a favor del pueblo hondureño. En el transcurso del evento, se compartieron emotivos discursos alusivos al homenaje (Figura 1).

Y surgen las preguntas ¿Dónde están los días de oro? ¿Vendrán los nuevos días? ${ }^{19}$ En la conmemoración del Aniversario 90 de la Revista Médica Hondureña, rendimos tributo a los médicos fallecidos durante la pandemia de $\frac{1930}{2}$ COVID-19 y extendemos este tributo a todo el personal de salud de Honduras y a sus hermosas y acongojadas familias.

\section{CONTRIBUCIONES}

JA concibió la idea, FM identificó y preparó la información, todos los autores contribuyeron al desarrollo del artículo y aprobaron su versión final.

\section{AGRADECIMIENTO}

Se agradece a la Lic. Martha Maradiaga y a la Dra. Helga I. Codina, Secretaría de Actas y Correspondencia, y a la Sra. Carolina Núñez y a la Lic. Jessie Carías, Gerencia Administrativa, Colegio Médico de Honduras, Tegucigalpa, por facilitar la información de los médicos fallecidos durante la pandemia de COVID-19.
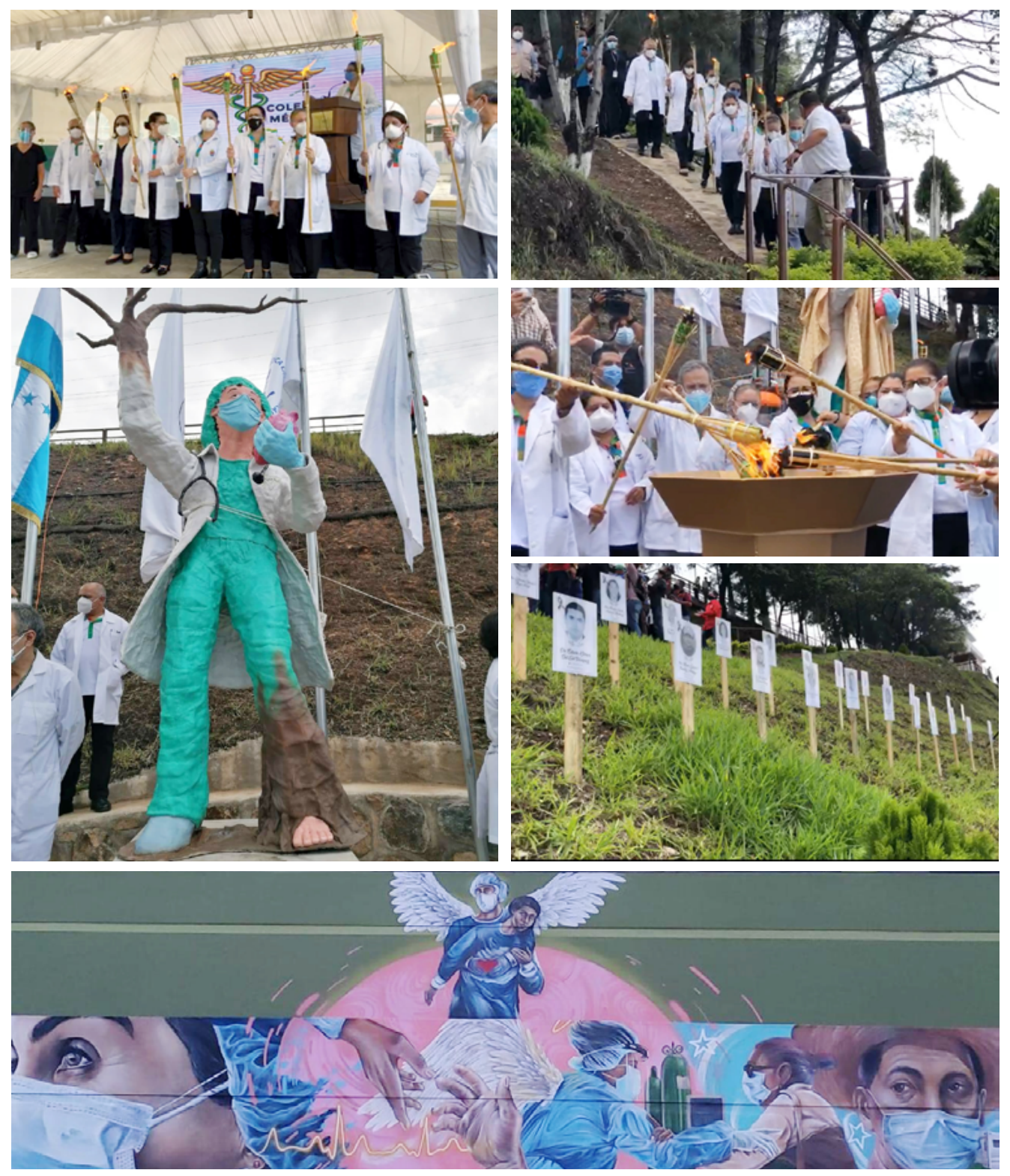

Figura 1. Homenaje a los médicos fallecidos durante la pandemia de COVID-19, Junta Directiva 2020-2022, Colegio Médico de Honduras, CA 6 Bulevar Fuerzas Armadas, Tegucigalpa, 22 de octubre de 2020. 


\section{REFERENCIAS}

1. Alger J [Editorial]. Revista Médica Hondureña: Aniversario 90 en tiempos de pandemia. Rev Méd Hondur. 2020; 88(1):7.

2. lyengar KP, Ish $P$, Upadhyaya GK, Malhotra N, Vaishya R, Jain VK. COVID-19 and mortality in doctors. Diabetes Metab Syndr: Clin Res Rev. 2020;14 (6):1743-746.

3. Pappa S, Ntella V, Giannakas T, Giannakoulis VG, Papoutsi E, Katsaounou P. Prevalence of depression, anxiety, and insomnia among healthcare workers during the COVID-19 pandemic: A systematic review and metaanalysis. Brain Behav Immun [Internet]. 2020[consultado 26 octubre 2020];88:901-907. Disponible en:https://doi.org/10.1016/j.bbi.2020.05.026

4. World Health Organization. WHO Director-General's opening remarks at the media briefing on COVID-19 - 11 March 2020. [Internet]. Ginebra: WHO; 2020. [consultado 26 octubre 2020]. Disponible en: https://www. who.int/director-general/speeches/detail/who-director-general-s-openingremarks-at-the-media-briefing-on-covid-19---11-march-2020

5. Worldometer. COVID-19 Coronavirus pandemic. [Internet]. S.I: worldometer; 2020. [consultado 26 octubre 2020]. Disponible en: https:// www.worldometers.info/coronavirus/

6. Johns Hopkins University. Coronavirus Resource Center. Mortality Analyses. [Internet].Maryland: Johns Hopkins University; 2020. [consultado 26 octubre 2020]. Disponible en: https://coronavirus.jhu.edu/data/mortality

7. Gobierno de Honduras. Despacho de Comunicaciones y Estrategia Presidencial. Línea de tiempo por día. [Internet]. Tegucigalpa: Despacho de Comunicaciones y Estrategia Presidencial; 2020. [consultado 26 octubre 2020]. Disponible en: https://covid19honduras.org/

8. Loewy MA. El dengue en Honduras es una tormenta perfecta epidemiológica. Medscape [Internet]. 28 de agosto de 2019. [consultado 26 octubre 2020]. Noticias y Perspectivas Disponible en: https://espanol. medscape.com/verarticulo/5904438

9. Codina HI [Editorial]. COVID-19 en Honduras. Rev Méd Hondur. 2020;
$88(1): 6$.

10. Banco Mundial. Honduras: panorama general. [Internet]: Tegucigalpa: Banco Mundial; 2020. [consultado 26 octubre 2020]. Disponible en: https:// www.bancomundial.org/es/country/honduras/overview

11. Trading Economics. Honduras military expenditure. [Internet].S.I: Trading econnomics; 2020. [consultado 26 octubre 2020]. Disponible en: https:// tradingeconomics.com/honduras/military-expenditure

12. Naciones Unidas. Comisión Económica para América Latina y el Caribe. América Latina y el Caribe ante la pandemia del COVID-19. Efectos económicos y sociales. Santiago de Chile: CEPAL; 2020.

13. Koh $D$ [Editorial]. Occupational risks for COVID-19 infection. Occup Med 2020;70:3-5.

14. Shah K, Chaudhari G, Kamrai D, Lail A, Patel RS. How essential is to focus on physician's health and burnout in coronavirus (COVID-19) Pandemic? Cureus 2020;12(4):e7538.

15. Klompas M. Coronavirus Disease 2019 (COVID-19): Protecting hospitals from the invisible. Ann Int Med. 2020;172(9):619-620.

16. Adams JG, Walls RM. Supporting the health careworkforce during the COVID-19 global epidemic. JAMA 2020;323(15):1439-1440.

17. COVID-19: protecting health-care workers [Editorial]. Lancet. 2020;395(102289):922.

18. World Health Organization. Keep health workers safe to keep patients safe: WHO [Internet]. Ginebra: WHO; 2020. [consultado 26 octubre 2020]. Disponible en: https://www.who.int/news/item/17-09-2020-keep-healthworkers-safe-to-keep-patients-safe-who

19. Guillén Zelaya A. Vendrán los nuevos días. [Internet]. Tegucigalpa: Red Honduras; 2020. [consultado 26 octubre 2020]. Disponible en: https:/l redhonduras.com/cultura/vendran-los-nuevos-dias-por-alfonso-guillenzelayal 